\title{
IMPROVING THE UPTAKE OF FLOOD RESILIENCE AT THE INDIVIDUAL PROPERTY LEVEL
}

\author{
CARLY ROSE ${ }^{1}$, JESSICA LAMOND ${ }^{1}$, MARY DHONAU ${ }^{2}$, ROTIMI JOSEPH $^{3} \&$ DAVID PROVERBS $^{4}$ \\ ${ }^{1}$ University of the West of England, UK. \\ ${ }^{2}$ MDA Community Flood Consultants, UK. \\ ${ }^{3}$ Cunningham Lindsey, UK. \\ ${ }^{4}$ Birmingham City University, UK.
}

\begin{abstract}
One of the drivers for improving resilience to flooding at property level (also known as adoption of a 'water entry strategy') was demonstrated by the overtopping of hard engineered flood defences across Cumbria, UK during 'Storm Desmond' in December 2015. Although the uptake of water exclusion strategies (also termed 'flood resistance) is gradually improving in the UK, the longer term resilience options that permit water entry are less popular. Findings from an evidence assessment of the barriers and drivers to uptake of low-cost water entry strategy options are presented. Evidence was collected using a Rapid Evidence Assessment of published materials, semi-structured interviews and workshops with professionals, and a series of case studies of properties that had been adapted to flood risk. Factors leading to successful implementation are identified and illustrated by case study material from homes and small businesses. Water entry and water exclusion approaches were found to be seen as two methods used as part of an integrated approach that can bring about important co-benefits such as improved thermal efficiency and lower energy bills.
\end{abstract}

Keywords: flooding, flood-proof, flood-repairable, resilience, water entry strategy.

\section{INTRODUCTION}

One of the drivers for improving resilience to flooding at property level (also known as adoption of a 'water entry strategy') was demonstrated by the overtopping of hard engineered flood defences across Cumbria, UK during 'Storm Desmond' in December 2015 [1]. The uptake of water exclusion strategies (also termed 'flood resistance) is gradually improving in the UK, in large part due to the demonstration projects funded by Defra and the Environment Agency between 2009 and 2012; the longer term resilience options that permit water entry, however, are less well known [2]. Water entry strategy has been defined [3] as:

Allow water through property to avoid risk of structural damage but noting that, for low depths, this strategy can also include Attempt to keep water out for low depths of flooding

Flood resilience within a building can be achieved in different ways: for example, avoidance of inundation by raising items such as electrical sockets above expected flood levels; or using resilient materials that do not deform/disintegrate on contact with floodwater, such as cementitious materials. In all cases, it is important to ensure that water can be expelled from the building quickly, and an adequate circulation of air around the exposed elements for reasonably rapid drying must be maintained. For any given building, therefore, there may be multiple possible water entry strategic solutions. 


\section{Flood Risk Management and Response}

Despite efforts by multiple agencies in the UK, the tendency of communities at risk to adopt measures to protect their property from flooding is generally low. Various aspects of flood adaptation behaviours have been studied [4-6]; while a study specific to 'resilient' or 'flood repairable' adaptation was conducted [7]. In each case, a complex set of constraints was found to exist that inhibited the necessary behavioural changes. In a review of the international literature on resilience, the findings are grouped in terms of four barrier types: informational, financial, emotional and timing issues [8]; these, in turn, impacted variously on components within the decision making process such as the necessary awareness and perception of risk; ownership of the risk; knowledge of solutions; resources to implement solutions (including financial issues); and belief that the measures would work. It has been found that, although resilient reinstatement did cost more than traditional repair, this approach did significantly reduce future repair costs (assuming a subsequent flood were to take place) [9].

The Department for the Environment, Food and Rural Affairs (Defra) in the UK therefore sought to more fully explore the research gap around the cost and performance of resilient measures [10]. The primary question for this research was formulated as:

'How can low-cost adaptation approaches be used in existing residential and small business properties to limit the damage from flood water'.

\section{METHOD}

First, a scoping exercise was undertaken, followed by consultation and interviews with key stakeholders. Informed by the findings from these stages, a Rapid Evidence Assessment (hereafter REA) of published material on resilience approaches was then conducted using a structured protocol, and encompassing both academic and 'grey' literature sources (such as industry/government publications and guidance). This initial work identified 89 resilience measures in all; costing information was, however, found to be scanty and contradictory in some cases, making it difficult to identify low-cost approaches definitively. Robust scientific evidence on actual performance of measures in flood events was also found to be lacking in many areas, although industry experts have consistently recommended a large number of the approaches concerned, based on their experiential evidence.

In the second phase, input from the professional and practitioner communities was gained by means of semi-structured interviews with 18 individuals. This method captured additional evidence about emerging approaches not represented in published sources, as well as examples of leading practice and reflection on the effectiveness of approaches. Potential literature sources identified by the respondents were located, where possible, and considered for inclusion in the final literature review. A second series of semi-structured interviews was conducted with 13 householders/small business owners, all of whom had already made adaptations to their properties. These interviews explored the experience of individuals at different stages in their adaptation journeys: the process of deciding to adapt; the adaptation activities themselves; and the learning points arising from living in their adapted property, particular the performance of different measures in subsequent flood events.

Third, an interim report summarising the outcomes of the above processes was submitted for critical appraisal by the industry experts who comprised the Project Board overseeing the research. This took the form of a workshop structured around key questions which would be used to inform the subsequent stages of the project. For example, an important issue was how 'low-cost' should be defined: for many of the measures, the financial outlay required could be 
very different if the work was undertaken as a part of the reinstatement process following flood damage, as opposed to the same work undertaken as a retrofitting option. This, in turn, generated many additional publications which were added to the REA database. The evidence collected from each of the householder/small business interviews were combined with photographic evidence (where available) to form summaries of the key learning points in a case study format.

\section{RESULTS}

The final list of measures identified numbered 139 in total and covered a wide range of water compatible building elements including: internal walls; flooring/floor coverings; kitchen and bathroom fixtures and fittings; various timber fixtures and fittings; approaches relating to building services and contents protection; and a miscellany of tried and tested hints and tips, largely provided by the householder and small business community. For example, less than $£ 40.00$ (48 Euros) is needed to purchase a 'sack truck' that allows items of furniture to be moved more easily when a flood warning has been issued. The review also highlighted the critical importance of understanding the specific detail of proposed measures: materials such as wallboards may bear superficially similar descriptions such as 'moisture resilient' or 'water resilient' but neither of these types is actually designed to be water-proof (boards designed to be fully water-proof exist, but can be considerably more expensive).

Where published studies included performance data, these largely reported the results of experiments on building assemblies, or building components subject to submersion or simulated flooding, on a limited number of materials. However, the properties of any material considered in isolation do not adequately predict resilience within a building setting: the nature and quality of construction, the interfaces between materials, and the drying spaces around them are equally relevant. Whilst there was anecdotal and testimonial evidence suggesting that a number of resilient interventions would be successful in preventing damage, the possibility of bias (towards the reporting of positive results) was likely to be present in sources such as this, as failures are unlikely to be reported.

Evidence of positive performance of some materials was provided by many of the professional interviewees, which largely concurred with current recommendations and is therefore unlikely to be subject to bias. The resilience of some materials traditionally subject to 'stripping out' was highlighted by some of these respondents: for example, several questioned the necessity of replacing (hardwood) timber floors with concrete for most properties. They also reported instances where normally resilient materials had deteriorated, usually after prolonged inundation. This group also emphasised the importance of considering building assemblies, joints and interfaces, rather than individual materials in isolation.

From the households and small business interviews, kitchens units made from both marinegrade plywood and plastic had been found to be resilient. Lime plaster, and sand and cement render, had been successfully used by some respondents. Here again, hardwood elements, together with (heavily) varnished and painted wood were reported to have survived repeated flood events, echoing the professionals' comments regarding the resilience of much timber, which calls into question the commonly accepted practice of 'stripping out' such elements. Households also reported on the success of some of the less frequently recommended measures, such as isolating ground floor circuits from those in the rest of the building (thereby allowing residents to live upstairs during and immediately after a flood); and, as well as moving contents, protecting items by, for example, wrapping in plastic, or using proprietary waterproof bags were recommended. Some issues were also reported, however, including 
concerns over sanitising contaminated kitchen surfaces; higher than expected flood depths; and failure under hydrostatic pressure. Other issues, such as minor salt accretion, compromised breathability and rusting issues with one steel kitchen appear to be related to existing building conditions rather than flood damage.

The most commonly reported factor that contributes to the desire to adapt property to flooding is flood experience, usually direct experience of flood damage to the property [11]. Householder interviews confirmed that their motivation for adaptation was typically triggered by the desire to avoid further flood damage, with its attendant distress and often lengthy displacement from their homes. It has been found this behavioural driver is strongest in the period immediately following a flood [12]; some professional interviewees, however, had noted a reluctance to slow down the reinstatement/reoccupation process by incorporating adaptations. This means that, even though adaptation has been found to be most cost effective at reinstatement [13] there may be a need to consider additional intervention points within the property life-cycle, such as pre-planned maintenance, or renewal of fixtures and fittings [14]. It has also been suggested that businesses, in particular, may be driven to install measures during insurance renewal [15]. General awareness campaigns, however, were not mentioned as a factor by any of the interviewees: this underscores the importance of utilising innovative awareness raising activities, targeted at the appropriate windows of opportunity.

There are a number of other issues directly impacting upon the most appropriate resilience choices for a specific property which were highlighted by some of the interviewees. These included the likely speed of onset, as surface water or 'flash' floods typically occur very rapidly and often lack any prior warning system; in such circumstances, passive measures represent the most appropriate solutions, as the building's occupants may be unable to put more planned measures into effect. This was reported by one householder, whose flood plan involved actions such as inserting proprietary toilet bungs, and removing internal doors; when the local flood warning system failed to operate late one night, the property was inundated far too rapidly for these measures to be deployed. The type and age of the property is also of importance: for example, historic buildings in the UK are typically subject to legal restrictions regarding alterations to the fabric of the structure, as well as its external appearance, which may mean making resilient alterations poses a greater challenge. Finally, the capacities and preferences of the building occupiers themselves must be taken into account: those with existing mobility issues may have difficulty deploying some measures, while aesthetic considerations, such as the desire to maintain the building's 'normal' appearance, cannot be disregarded.

\section{CASE STUDIES}

Most of the householders had been flooded more than once, with the worst case being 14 times in 12 years. For some, however, a single flood, or knowledge of the flood risk, had prompted them to install measures; these individuals appear to be atypical and more proactive than the average population at risk, as $6 \%$ is the typical proportion found to act in these circumstances [16]. The commonest adaptation among the householders interviewed was the raising of electrical sockets above the anticipated flood levels, with the supply cables running down from above. Tiled floors were frequently adopted (including ceramic, porcelain, reconstituted marble and encaustic); one had chosen solid wood flooring that was 'heavily varnished' (the latter having already survived three inundations unscathed), while another had selected very cheap (sacrificial) carpet tiles to cover a concrete floor. In other respects, however, both the variety and ingenuity of adaptation measures was remarkably wide ranging. 


\subsection{Case Study 1 - Worcestershire, UK}

The first case concerns a property with extensive and comprehensive adaptations, being an 18th century half-timbered cottage which had suffered fluvial flooding twice during the owner's period of occupancy. On the first occasion around $20 \mathrm{~cm}$ of water entered the building causing some minor damage and an insurance claim was made. Seven years later, a major flood occurred, with over $1 \mathrm{~m}$ of water inside the house: some structural damage resulted, and the owner was advised that extensive rebuilding work would be required. This prompted the owner to investigate what resilience measures could be incorporated during this process, not only to avoid having to go through the 'upheaval and trauma' again, but also due to wellfounded fears that insurance cover would become prohibitively expensive in the future.

In addition to many of the common solutions shown in Fig. 1, some innovative measures have been adopted: for example, in the kitchen the dishwasher is not permanently plumbed in, so that it can easily be removed to a safer location when flooding is forecast. An extension to the oiled oak work surface forms a breakfast bar supported by stainless steel legs (as opposed to the more usual chipboard or MDF end panel); the accompanying bar stools are an elegant Italian design, but are made of fully washable plastic material. In the lounge, the owner's extensive book collection is accommodated on shelving supported by wall brackets (rather than traditional free standing bookcases); furthermore, the lowest shelves are easily removable, once the books themselves have been packed away and moved to safety. Many items of furniture on the ground floor are of a lightweight design so they can easily be moved upstairs upon receipt of a flood warning. Long-term sustainability was also an important factor for the owner, with the central heating radiators being not only removable, but also the most energy-efficient design available, to minimise future heating bills. In order to reoccupy the property as soon as possible following a future flood, the upstairs rooms are now on

The Flood-Repairable House

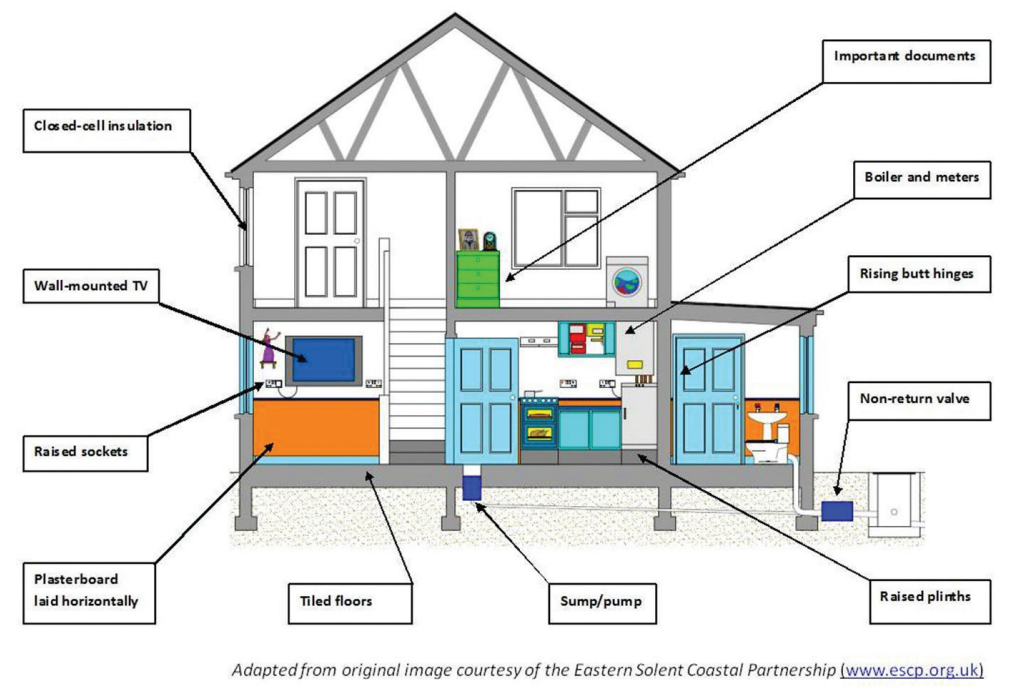

Figure 1: The Flood Repairable House (as adapted from original image, courtesy of the Eastern Solent Coastal Partnership - www.escp.org.uk). 
separate circuits from the ground floor: this allows the owner to live in relative comfort upstairs, with heating and lighting unaffected, while the ground floor rooms are being dried out and cleaned.

The owner in this instance had undertaken a considerable amount of research via the Internet in order to identify the most appropriate solutions in costing terms: for example, an open tread style of solid wood staircase was selected, as this cost the same as the standard design made of inferior quality materials, a change that was, therefore, cost neutral to the insurance company. The replacement kitchen units chosen were of powder-coated steel, as this was not only water resilient, but also had an advertised life-span of around 30 years, which the owner felt justified the higher initial outlay. This property, therefore, includes several measures that have co-benefits, as well as meeting the needs of the owner in terms of aesthetic acceptability.

\subsection{Case Study 2 - Buckinghamshire, UK}

A four-floor 19th century semi-detached property situated in a town with a long history of flooding. The owner was not aware of the flood risk at the point of purchase, however, and as part of the initial renovation work converted the basement level to create a living room and a hi-tech kitchen. The property subsequently suffered two floods within 5 years, and the owner (belatedly) realised the appropriateness of the original Victorian design, in which the basement had housed the servants' quarters, with stone-flagged floors laid on sand. This had, in effect, been flood resilient as water was allowed in, but it also had an escape route following a flood. After the second flood event the owner, who had some practical building experience, then decided to make the house resilient, using what they described as a 'common-sense approach'.

The kitchen floor was re-covered using encaustic tiles affixed with waterproof cement, which can be easily washed down. The kitchen units were constructed from marine ply, which can also be washed out and disinfected; removable kickboards permit the voids below to dry out post flooding. A sump and pump installation is designed to keep the water level within the property as low as possible, and the electricity supply for this comes from the separately zoned upper floor. The free-standing items of furniture in the affected rooms have been selected to be easily raised on stacks of bricks; as a variation on this common method; however, in this case, the bricks are wrapped in plastic bags, to prevent water permeating through bricks and thus affecting the wooden legs of the furniture.

The owner of this property had successfully applied for a government grant to cover the costs of some of the changes made following the second flood, but, as with Study 1, they too had carefully considered the long-term resilient options they wished to adopt. For example, using marine ply to construct kitchen units involves a relatively high initial layout, but was expected to repay the cost in the course of subsequent flood events.

\subsection{Case Study 3 - Yorkshire}

A small Victorian property which the owner has rented to the current tenant for four years, during which period four 'flash 'floods have occurred (typically with very little warning time, owing to the steepness of the local catchment). One of the reasons for opting for a resilient approach is that the occupant has several pets, making it difficult to obtain alternative accommodation following flooding (as many landlords will not allow animals in their properties). Both parties agreed upon a programme of alterations that was acceptable both in cost terms (to the owner) and on aesthetic and practical terms (to the tenant). As with Study 1, the house 
has been rewired with a separate circuit serving the upper floor, enabling the occupant to continue living in the property while the lower floors are being dried and cleaned. A modestly priced (and therefore sacrificial) carpet has been used to cover the concrete floor of the living room, but the tenant uses a good quality, easily removable rug on top of this. As the original front door had been coming towards the end of its useful life, it was replaced by a low maintenance and draught-free UPVC version, benefitting both the tenant and property owner alike. Finally, the tenant chose to change the way in which some of the rooms were used, including ceasing to store items in the cellar.

\section{DISCUSSION}

There seemed to be a consensus, from both professionals and householders, that the water entry approach is about damage limitation, with water exclusion strategies remaining the preferred option. Respondents from both groups favoured a combination of measures to limit water entry, as well as incorporating repairable internal features. This suggests that repairable approaches might usefully be reappraised: instead of being seen as the alternative of last resort, adoption could be promoted as being useful within any property level scheme. Stressing co-benefits could be helpful when recommending adaptations: for example, the householder who had purchased stylish Italian furniture, enhanced her interior décor, as well as ensuring the items were lightweight and resilient. Similarly, raised sockets can be convenient in the longer term, as they are more easily accessed by older people, or those with mobility issues; both waterproof insulation materials and UPVC door and window frames offer improved thermal efficiency and, thus, lower energy bills.

\section{CONCLUSIONS}

The weight of evidence suggests that low-cost approaches can be used to prevent some of the damage from floodwaters entering the home and increasing their uptake could save money for households, small businesses and their insurers. Of the 138 measures identified, over 70 were deemed to be 'low-cost' or 'affordable', depending upon the point in the property life-cycle when the adaptations are made (for example, during post-flood repair work, or as part of other property renovations or repairs). Although scientific evidence of performance is lacking in some cases, industry experts have consistently recommended many of the approaches examined; more research is, therefore, needed to gather improved evidence of effectiveness. This will need to combine laboratory testing, testimonial and documentary evidence and post flood damage assessments, not only of individual materials, but with multiple components as used in building assemblies. The majority of the householder interviewees, and many professionals, emphasised that more information and guidance was needed in this area, supporting the view that overcoming informational barriers will be critical in improving uptake. Although the extant literature and guidance often make a binary distinction between water entry and water exclusion, interviewees saw the two methods as forming important elements of an integrated approach.

\section{ACKNOWLEDGEMENT}

This research is funded by Defra under project reference FD2682.

\section{REFERENCES}

[1] Balmforth, D., Why did flood defences fail against Storm Desmond? 201516 December, 2015 [cited 2015; available at: http://www.ice.org.uk/media-and-policy/the-infrastructure-blog/december-2015/why-did-flood-defences-fail-against-storm-desmond. 
160 Flood Risk Management and Response

[2] JBA Consulting, Establishing the Cost Effectiveness of Property Flood Protection: FD2657 - Final Report, Defra: London, 2013.

[3] Tagg, A., Escarameia, M. \& Ortiz, J.M., Improving the flood resilience of buildings through improved materials, methods and details - Report WP6 - collation and analysis of post-flood observational data, in DCLG Building Regulations (Sanitation) Framework. 2007.

[4] Thurston, N., Finlinson, B., Breakspear, R., Williams, N., Shaw, J. \& Chatterton, J., Developing the Evidence Base for Flood Resistance and Resilience - R\&D Technical Report FD2607/TR, Defra: London. p. 24, 2008.

[5] Spence, A., Poortinga, W., Butler, C. \& Pidgeon, N.F., Perceptions of climate change and willingness to save energy related to flood experience. Nature Climate Change, $\mathbf{1}$, pp. 46-49, 2011.

http://dx.doi.org/10.1038/nclimate1059

[6] Bubeck, P., Botzen, W.J.W. \& Aerts, J.C.J.H., A review of risk perceptions and other factors that influence flood mitigation behavior. Risk Analysis, 32(9), pp. 1481-1495, 2012. http://dx.doi.org/10.1111/j.1539-6924.2011.01783.x

[7] Wassell, P., Ayton-Robinson, R., Robinson, D., Joseph, R., Hack, K., Butler, D., et al., Resilient Reinstatement - the Cost of Flood Resilient Reinstatement of Domestic Properties (ABI Research Paper no 14, 2009). In Research Paper, Association of British Insurers: London, 2009.

[8] Lamond, J.E. \& Proverbs, D.G., Resilience to flooding: lessons learned from an international comparison. Proceedings of the Institution of Civil Engineers - Urban Design and Planning, 162(2), pp. 63-70, 2009. http://dx.doi.org/10.1680/udap.2009.162.2.63

[9] Joseph, R., Proverbs, D., Lamond, J. \& Wassell, P., An analysis of the costs of resilient reinstatement of flood affected properties. Structural Survey, 29(4), pp. 279-293, 2011. http://dx.doi.org/10.1108/02630801111162350

[10] Defra. Low Cost Resilience - FD2682. 2015, available at: http://sciencesearch.defra. gov.uk/Default.aspx $?$ Menu=Menu\&Module $=$ More $\&$ Location $=$ None $\&$ Completed $=0 \&$ ProjectID=19221.

[11] Rose, C.B., Proverbs, D.G., Booth, C.A. \& Manktelow, K.I., Three times is enemy action - flood experiences and flood perceptions. In: FRIAR III (3rd International Conference on Flood Recovery, Innovation and Response), eds. D. Proverbs, S. Mambretti, C.A. Brebbia \& D. de Wrachien, WIT Press: Dubrovnik, Croatia. pp. 233-242, 2012.

[12] Steinführer, A., Local communities at risk from flooding: social vulnerability, resilience and recommendations for flood risk management in Europe, Helmholtz centre for environmental research - UFZ Leipzig, 2009.

[13] Joseph, R., Proverbs, D. \& Lamond, J., Resilient reinstatement: what can we learn from the 2007 flooding in England? Flood Recovery, Innovation and Response IV, 184, p. $175,2014$.

[14] Soetanto, R., Proverbs, D.G., Lamond, J. \& Samwinga, V., Residential properties in England and Wales: an evaluation of repair strategies towards attaining flood resilience. In Hazards and the Built Environment: Attaining Built-in Resilience, ed. L. Boscher, Taylor and Francis, pp. 124-149, 2008.

[15] Lamond, J., Dhonau, M., Rose, C. \& Proverbs, D., Overcoming the barriers to installing property level flood protection - an overview of successful case studies. In Road Map 
Towards a Flood Resilient Urban Environment, Urban Flood Management Cost Action Network C22: Paris, 2009.

[16] Harries, T., Public perceptions and adaptations to flood risk. In Flood Repair Network Workshop no 4, 08/05/08, ed. D.G. Proverbs, Embassy House: Birmingham UK, 2008. 